KANAZAWA-14-08

September, 2014

\title{
Inflation in a modified radiative seesaw model
}

\author{
Romy H. S. Budhi 1,2:, Shoichi Kashiwase 2, \\ and \\ Daijiro Suematsu $2:+$ \\ ${ }^{1}$ Physics Department, Gadjah Mada University, Yogyakarta 55281, Indonesia \\ ${ }^{2}$ Institute for Theoretical Physics, Kanazawa University, Kanazawa 920-1192, Japan
}

\begin{abstract}
The existence of the inflationary era in the early Universe seems to be strongly supported by recent CMB observations. However, only a few realistic inflation scenarios which have close relation to particle physics seem to have been known unfortunately. The radiative neutrino mass model with inert doublet dark matter is a promising model for the present experimental issues which cannot be explained within the standard model. In order to make the model include inflation, we extend it by a complex scalar field with a specific potential. This scalar could be closely related to the neutrino mass generation at a $\mathrm{TeV}$ scale as well as inflation. We show that the inflation favored by the CMB observations could be realized even if inflaton takes sub-Planck values during inflation.
\end{abstract}

\footnotetext{
*e-mail: romyhanang@hep.s.kanazawa-u.ac.jp

${ }^{\dagger}$ e-mail: shoichi@hep.s.kanazawa-u.ac.jp

$\ddagger$ e-mail: suematsu@hep.s.kanazawa-u.ac.jp
} 


\section{Introduction}

Recent discovery of a Higgs-like particle [1] suggests that the framework of the standard model (SM) can describe Nature well up to the weak scale. On the other hand, we have experimental results which cannot be explained within it, that is, the existence of small neutrino masses [2, 3], the existence of dark matter [4], and baryon number asymmetry in the Universe [5]. They require some extension of the SM.

As such an example, we have a model which is the simple extension of the SM with a second doublet scalar (which has no vacuum expectation value and is called by several names such as inert [6], scotogenic [7], or doumant [8]) and also three right-handed neutrinos. The model shows promising features in physics at $\mathrm{TeV}$ regions for the explanation of both the neutrino oscillation data and the observed abundance of dark matter (DM). In fact, if these new fields are assigned odd parity of an assumed $Z_{2}$ symmetry, small neutrino masses are generated at one-loop level and the lightest $Z_{2}$ odd field can be stable as a DM candidate [9]. The quantitative conditions required for their explanation in both this model and several extended models have been clarified through various studies by now [10, 11, 12, 13, 14]. They show that the simultaneous explanation of these is possible without causing a strong tension with other phenomena like lepton flavor violating processes if DM is identified with the lightest neutral component of the inert doublet scalar [11, 14]. In such a case, moreover, the baryon number asymmetry in the Universe is also

successfully explained if the resonant leptogenesis could occur due to the mass degeneracy among right-handed neutrinos which have masses of a TeV scale [15]. An interesting point is that the required mass degeneracy could be rather mild compared with the ordinary cases [14].

The CMB observations suggest that the exponential expansion of the Universe occurs in the very early Universe. These results can constrain severely the allowed inflation models now [16, 17]. For example, BICEP2 recently suggests that the tensor to scalar perturbation ratio should be $r \sim 0.2$ and the Hubble parameter during inflation should take a value of $O\left(10^{14}\right) \mathrm{GeV}$. Although we know that a quadratic chaotic inflation model could be such a candidate, the inflaton should take trans-Planckian values during inflation in that model. Since higher order terms which are suppressed by the Planck mass are generally expected to give larger contributions to the potential there, the flatness of potential cannot be guaranteed without any symmetry. 
On the other hand, we do not have a lot of examples of inflaton that plays any role in particle physics. Inflaton is introduced just to bring about the inflation in many models. As an exceptional example, one may suppose sneutrino inflation [18] al If we consider the neutrino mass generation based on the seesaw mechanism in supersymmetric models, right-handed sneutrinos are introduced inevitably. One of them could work as inflaton causing the quadratic chaotic inflation. However, the model could be annoyed by the above mentioned trans-Planckian problem.

In this paper, we propose an inflation scenario in the framework related to the radiative seesaw model. Although several inflation scenarios have been considered in the radiative seesaw model, they have still problems, for example, the above mentioned trans-Planckian problem [13] or the unitarity problem caused by a large non-minimal coupling [21]. Our scenario is based on an extension of the radiative seesaw model with a complex scalar, whose component is identified with the inflaton. We show that sufficient e-foldings could be realized even if the inflaton takes sub-Planckian values during inflation. In this scenario, the scalar spectral index and the tensor-to-scalar ratio could have values in the region favorable from the recent precise CMB observations. In particular, the tensor-to-scalar ratio could take rather wide range values consistent with the CMB results depending on the parameters in the inflaton potential. Moreover, this inflaton could play a crucial role for the neutrino mass generation other than the inflation, which is similar to the sneutrino inflation scenario.

The paper is organized as follows. In the next section we address our extended model briefly. In particular, the role of a new singlet scalar in the neutrino mass generation is explained. In section 3, we study the inflation in this model. Important quantities relevant to the inflation such as e-foldings, slow-roll parameters and spectral index are estimated numerically. Reheating temperature is also discussed. In section 4 we summarize the paper.

\footnotetext{
${ }^{\text {a} H i g g s ~ i n f l a t i o n ~[19] ~ a n d ~ a x i o n i c ~ i n f l a t i o n ~[20] ~ a r e ~ a l s o ~ m o t i v a t e d ~ b y ~ p a r t i c l e ~ p h y s i c s . ~}$
} 


\section{$2 \quad$ An extended model}

The original radiative seesaw model is defined by the following $Z_{2}$ invariant terms [9]:

$$
\begin{aligned}
-\mathcal{L}_{O} & =-h_{\alpha i} \bar{N}_{i} \eta^{\dagger} \ell_{\alpha}-h_{\alpha i}^{*} \bar{\ell}_{\alpha} \eta N_{i}+\frac{M_{i}}{2} \bar{N}_{i} N_{i}^{c}+\frac{M_{i}^{*}}{2} \bar{N}_{i}^{c} N_{i} \\
& +m_{\phi}^{2} \phi^{\dagger} \phi+m_{\eta}^{2} \eta^{\dagger} \eta+\lambda_{1}\left(\phi^{\dagger} \phi\right)^{2}+\lambda_{2}\left(\eta^{\dagger} \eta\right)^{2}+\lambda_{3}\left(\phi^{\dagger} \phi\right)\left(\eta^{\dagger} \eta\right)+\lambda_{4}\left(\eta^{\dagger} \phi\right)\left(\phi^{\dagger} \eta\right) \\
& +\frac{1}{2}\left[\lambda_{5}\left(\eta^{\dagger} \phi\right)^{2}+\lambda_{5}^{*}\left(\phi^{\dagger} \eta\right)^{2}\right],
\end{aligned}
$$

where $\ell_{i}$ is a left-handed lepton doublet and $\eta$ is an inert doublet scalar. Since $\eta$ and righthanded neutrinos $N_{i}$ are assigned odd parity of $Z_{2}$ symmetry and all the SM contents including the ordinary Higgs doublet scalar $\phi$ are assigned even parity, neutrino Dirac mass terms are forbidden at tree-level. Neutrino masses are generated through one-loop diagram which has $N_{i}$ and $\eta$ in the internal lines as shown in the left diagram of Fig. 1.

As found from this figure, neutrino mass generation in this model is characterized by a scalar quartic coupling $\lambda_{5}\left(\eta^{\dagger} \phi\right)^{2}$ between the ordinary doublet Higgs scalar $\phi$ and an inert doublet scalar $\eta$. In this mass generation at $\mathrm{TeV}$ scales, the smallness of the coupling $\lambda_{5}$ plays a crucial role to explain the smallness of neutrino masses. It is considered to be a key feature of this scenario. To explain its smallness, we may consider a scenario that this coupling is an effective coupling appearing at low energy regions after integrating out a heavy complex singlet scalar $S$. Such a scenario could be realized by introducing a $Z_{2}$ odd singlet complex scalar. Additional terms in the new Lagrangian are given as

$$
\begin{aligned}
-\mathcal{L}_{S} & =\tilde{m}_{S}^{2} S^{\dagger} S+\frac{1}{2} m_{S}^{2} S^{2}+\frac{1}{2} m_{S}^{2} S^{\dagger 2}+\kappa_{1}\left(S^{\dagger} S\right)^{2}+\kappa_{2}\left(S^{\dagger} S\right)\left(\phi^{\dagger} \phi\right)+\kappa_{3}\left(S^{\dagger} S\right)\left(\eta^{\dagger} \eta\right) \\
& -\mu S \eta^{\dagger} \phi-\mu^{*} S^{\dagger} \phi^{\dagger} \eta,
\end{aligned}
$$

where these are most general terms which are $Z_{2}$ invariant and renormalizable $\mathrm{b}$

If the new singlet $S$ is much heavier than $\eta$ and $N_{i}$, favorable features of the original model could be kept in this extended version. Neutrino mass is generated through the one-loop diagram shown in the right one of Fig. 1. In this diagram, $\varphi_{a}$ represents the component fields of $S$ which are defined as $S=\frac{1}{\sqrt{2}}\left(\varphi_{1}+i \varphi_{2}\right)$. Their masses are easily found to be $\bar{m}_{1}^{2}=\tilde{m}_{S}^{2}+m_{S}^{2}$ and $\bar{m}_{2}^{2}=\tilde{m}_{S}^{2}-m_{S}^{2}$. Since $Z_{2}$ is considered as an exact symmetry, $\tilde{m}_{S}^{2}>m_{S}^{2}$ should be satisfied. The similar diagram is know to contribute

\footnotetext{
${ }^{\mathrm{b}}$ In this extension, $\lambda_{5}=0$ is supposed in eq. (11). Since the $\beta$-function of $\lambda_{5}$ is proportional to $\lambda_{5}$ itself, this assumption is justified after taking into account the radiative correction.
} 

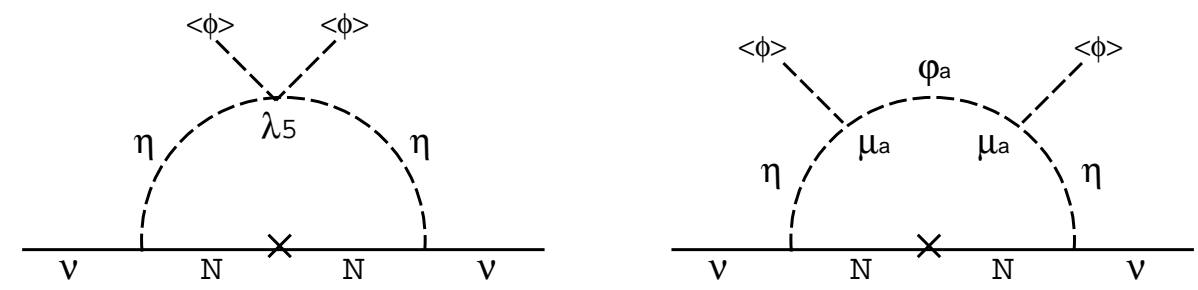

Fig. 1 One-loop diagrams which contribute neutrino mass generation. Left one is the diagram in the original model. The right diagram generates neutrino masses in the present model. The dimensionful coupling $\mu_{a}$ is defined as $\mu_{1}=\frac{\mu}{\sqrt{2}}$ and $\mu_{2}=\frac{i \mu}{\sqrt{2}}$ by using $\mu$ in eq. (2).

to the neutrino mass generation in the supersymmetrized radiative seesaw model [12]. Neutrino mass induced through this diagram can be estimated as

$$
\left(\mathcal{M}_{\nu}\right)_{\alpha \beta}=\sum_{i=1}^{3} \sum_{a=1,2} \frac{h_{\alpha i} h_{\beta i} M_{i} \mu_{a}^{2}\langle\phi\rangle^{2}}{8 \pi^{2}} I\left(M_{\eta}, M_{i}, \bar{m}_{a}\right)
$$

where $M_{\eta}^{2}=m_{\eta}^{2}+\left(\lambda_{3}+\lambda_{4}\right)\langle\phi\rangle^{2}$ and $\mu_{a}$ is defined as $\mu_{1}=\frac{\mu}{\sqrt{2}}$ and $\mu_{2}=\frac{i \mu}{\sqrt{2}}$, respectively. The function $I\left(m_{a}, m_{b}, m_{c}\right)$ is defined as

$$
\begin{aligned}
I\left(m_{a}, m_{b}, m_{c}\right) & =\frac{\left(m_{a}^{4}-m_{b}^{2} m_{c}^{2}\right) \ln m_{a}^{2}}{\left(m_{b}^{2}-m_{a}^{2}\right)^{2}\left(m_{c}^{2}-m_{a}^{2}\right)^{2}}+\frac{m_{b}^{2} \ln m_{b}^{2}}{\left(m_{c}^{2}-m_{b}^{2}\right)\left(m_{a}^{2}-m_{b}^{2}\right)^{2}} \\
& +\frac{m_{c}^{2} \ln m_{c}^{2}}{\left(m_{b}^{2}-m_{c}^{2}\right)\left(m_{a}^{2}-m_{c}^{2}\right)^{2}}-\frac{1}{\left(m_{b}^{2}-m_{a}^{2}\right)\left(m_{c}^{2}-m_{a}^{2}\right)}
\end{aligned}
$$

If we assume that the conditions $\tilde{m}_{S} \gg m_{S}, m_{\eta}, M_{i}$ are satisfied, this formula can be approximated as

$$
\left(\mathcal{M}_{\nu}\right)_{\alpha \beta}=\sum_{i=1}^{3} \frac{h_{\alpha i} h_{\beta i}\langle\phi\rangle^{2}}{8 \pi^{2}} \frac{m_{S}^{2} \mu^{2}}{\tilde{m}_{S}^{4}} \frac{M_{i}}{M_{\eta}^{2}-M_{i}^{2}}\left[\frac{M_{i}^{2}}{M_{\eta}^{2}-M_{i}^{2}} \ln \frac{M_{i}^{2}}{M_{\eta}^{2}}+1\right]
$$

It is equivalent to the neutrino mass formula in the original model if $\frac{m_{S}^{2} \mu^{2}}{\tilde{m}_{S}^{4}}$ is identified with the coupling constant $\lambda_{5}$ for the $\left(\eta^{\dagger} \phi\right)^{2}$ term.

This correspondence might be found in an effective theory obtained at energy regions smaller than $\tilde{m}_{S}$ by integrating out $S$. In fact, if we use the equation of motion for $S$ which could be approximated as $S \simeq \mu^{*} \phi^{\dagger} \eta / \tilde{m}_{S}^{2}$, the required terms are derived as

$$
\frac{1}{2}\left[\frac{m_{S}^{2} \mu^{2}}{\tilde{m}_{S}^{4}}\left(\eta^{\dagger} \phi\right)^{2}+\frac{m_{S}^{2} \mu^{* 2}}{\tilde{m}_{S}^{4}}\left(\phi^{\dagger} \eta\right)^{2}\right] .
$$

The origin of small $\lambda_{5}$ which is the key nature to explain the smallness of the neutrino masses is now translated to the hierarchy problem between $\mu, m_{S}$ and $\tilde{m}_{S}$ in this extension. 
If we leave the origin of this hierarchy to a complete theory at high energy regions, all the neutrino masses, the DM abundance and the baryon number asymmetry could be also explained in this extended model at $\mathrm{TeV}$ regions just as in the same way discussed in the previous articles [14]. Following the results obtained in these studies, the value of $\frac{m_{S}^{2} \mu^{2}}{\tilde{m}_{S}^{2}}$ could be constrained by the simultaneous explanation of these.

\section{Inflation due to the complex scalar $S$}

\section{1 e-foldings and the spectral index}

If the singlet scalar $S$ does not play any other role, this modification might not be so interesting. However, we find that the introduction of $S$ could add an interesting feature to the radiative seesaw model as an inflation model $c$ As such simple scenarios for a real singlet scalar $S$, one may consider $m_{S}^{2} S^{2}$ type chaotic inflation [13] or $S$-inflation [22]. In the former example, the inflation could be related with the neutrino mass generation like sneutrino inflaton model. However, the scenario requires trans-Planckian values for $S$ during inflation and it could induce the above mentioned problem.

In this section, we consider an inflation scenario which could work even for subPlanckian values of $S$, following the proposal in [23]. We show that it is possible as long as the existence of specific nonrenormalizable terms is assumed in the potential for $S$. As such potential, we suppose that the complex scalar $S$ has $Z_{2}$ invariant additional potential terms such as

$$
\begin{aligned}
V & =c_{1} \frac{\left(S^{\dagger} S\right)^{n}}{M_{\mathrm{pl}}^{2 n-4}}\left[1+c_{2}\left\{\left(\frac{S}{M_{\mathrm{pl}}}\right)^{2 m} \exp \left(i \frac{S^{\dagger} S}{\Lambda^{2}}\right)+\left(\frac{S^{\dagger}}{M_{\mathrm{pl}}}\right)^{2 m} \exp \left(-i \frac{S^{\dagger} S}{\Lambda^{2}}\right)\right\}\right] \\
& =c_{1} \frac{\varphi^{2 n}}{2^{n} M_{\mathrm{pl}}^{2 n-4}}\left[1+2 c_{2}\left(\frac{\varphi}{\sqrt{2} M_{\mathrm{pl}}}\right)^{2 m} \cos \left(\frac{\varphi^{2}}{2 \Lambda^{2}}+2 m \theta\right)\right],
\end{aligned}
$$

where both $n$ and $m$ are positive integers and $M_{\mathrm{pl}}$ is the reduced Planck mass. We use the polar coordinate expression $S=\frac{\varphi}{\sqrt{2}} e^{i \theta}$ in the second equality of eq. (17). Although the exponential part of this potential is crucial for the present scenario, we cannot describe its origin concretely at the present stages. We only expect that it might be effectively induced through the nonperturbative dynamics in the UV completion of the model. However, since

\footnotetext{
${ }^{\mathrm{c}}$ Higgs inflation has been applied to the radiative seesaw model in [21.
} 

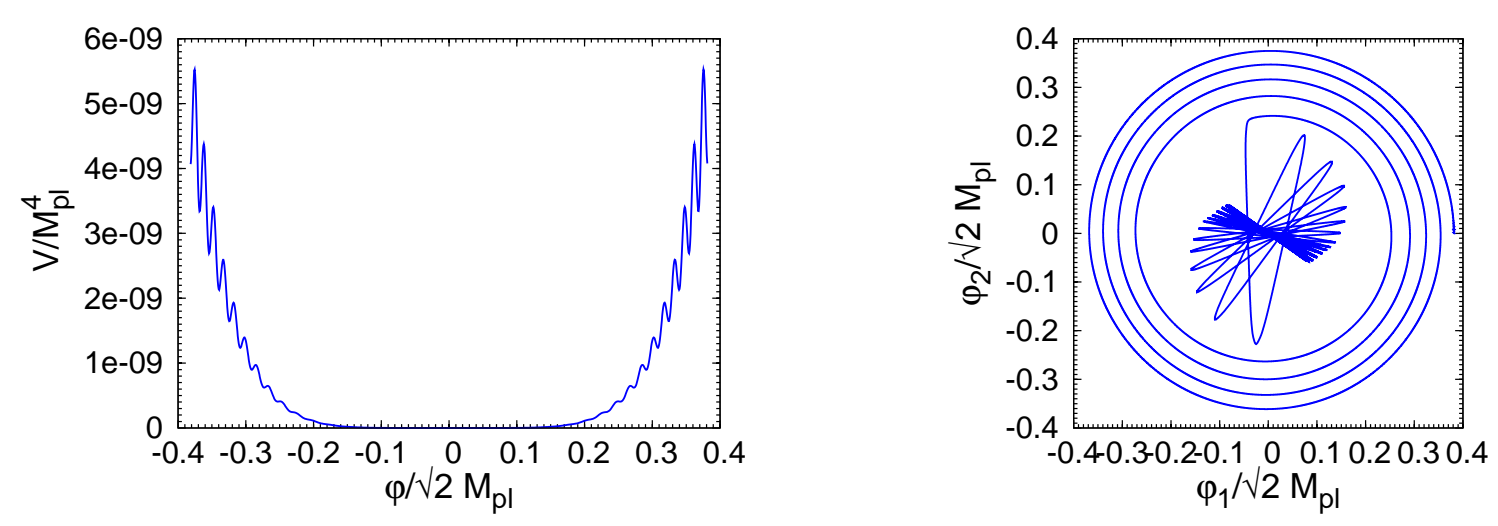

Fig. 2 The left panel shows the potential $V$ defined by $n=3$ and $m=1$. Other parameters in $V$ are fixed as $c_{1}=1.65 \times 10^{-6}, c_{2}=0.7$ and $\Lambda / M_{\mathrm{pl}}=0.04$. In the right panel, the time evolution of the field $a$ in the $\left(\frac{\varphi_{1}}{\sqrt{2} M_{\mathrm{pl}}}, \frac{\varphi_{2}}{\sqrt{2} M_{\mathrm{pl}}}\right)$ plane for the potential $V$ shown in the left panel. $\varphi$ is related to $\varphi_{1,2}$ by $\varphi^{2}=\varphi_{1}^{2}+\varphi_{2}^{2}$.

the model has interesting features for the inflation as shown below, we expect that the potential form might give us some useful hints to find the UV completion and its dynamics.

In the left panel of Fig. 2, we show a typical shape of the potential as a function of $\varphi$ for a fixed $\theta$. As easily found, the potential $V$ has local minimums for a fixed $\theta$ under the condition $\Lambda \ll \varphi \ll M_{\mathrm{pl}}$ at

$$
\frac{\varphi^{2}}{2 \Lambda^{2}}+2 m \theta=(2 j+1) \pi+\alpha \simeq(2 j+1) \pi
$$

where $j$ is an integer and $\alpha=\tan ^{-1}\left(\frac{2 \Lambda^{2}}{M_{\mathrm{pl}}^{2}}\right)$.

Now we assume that inflation proceeds along this local minimum. In that case, the field $a$ along this direction is considered to play a role of inflaton. It might be represented as $d a^{2}=d \varphi^{2}+\varphi^{2} d \theta^{2}$. Since $\varphi$ is supposed to evolve as a function of $\theta$ following eq. (8), we find that the field $a$ should satisfy the relation such as

$$
d a=\left[\varphi^{2}+\left(\frac{d \varphi}{d \theta}\right)^{2}\right]^{1 / 2} d \theta=\left[1+4 m^{2}\left(\frac{\Lambda}{\varphi}\right)^{4}\right]^{1 / 2} \varphi d \theta
$$

This shows that the field $a$ can be expressed as $d a \simeq \varphi d \theta$ as long as $\varphi \gg \Lambda$ is satisfied. Thus, the field $a$ associated to the almost flat direction can be treated as a canonically normalized inflaton field orthogonal to $\varphi$. In order to estimate the mass of $\varphi$ during the period when the field evolve along the $a$ direction, we expand the potential $V$ given in eq. (77) around its local minimum at a fixed $\theta$. As this result, we find that the mass of $\varphi$ 
satisfies

$$
m_{\varphi}>\left[\frac{c_{1}}{2^{n-1}} n(2 n-1)\right]^{1 / 2}\left(\frac{\varphi}{M_{\mathrm{pl}}}\right)^{n-2} \varphi .
$$

On the other hand, the Hubble parameter during this period could be roughly estimated as

$$
H=\left(\frac{V}{3 M_{\mathrm{pl}}^{2}}\right)^{1 / 2} \simeq\left(\frac{c_{1}}{3 \cdot 2^{n}}\right)^{1 / 2}\left(\frac{\varphi}{M_{\mathrm{pl}}}\right)^{n-1} \varphi
$$

As long as we suppose a situation such as $\varphi \ll M_{\mathrm{pl}}$, we find that the mass of $\varphi$ is much larger than the Hubble parameter there. This shows that $\varphi$ cannot contribute to the inflation and then the single inflaton scenario due to the field $a$ could be realized.

We can check that this actually occurs through numerical calculation. We solve the field equations for the component fields $\varphi_{1,2}$ of $S=\frac{1}{\sqrt{2}}\left(\varphi_{1}+i \varphi_{2}\right)$ numerically. They evolve following the field equations,

$$
\ddot{\varphi}_{i}+3 H \dot{\varphi}_{i}=-\frac{\partial V}{\partial \varphi_{i}} \quad(i=1,2)
$$

where $H^{2}=\frac{1}{3 M_{\mathrm{pl}}^{2}}\left(\sum_{i} \frac{1}{2} \dot{\varphi}_{i}^{2}+V\right)$. For example, the explicit expression for $\frac{\partial V}{\partial \varphi_{i}}$ in the case of $n=3$ and $m=1$ is given as

$$
\begin{aligned}
\frac{\partial V}{\partial \varphi_{1}} & =\frac{c_{1}\left(\varphi_{1}^{2}+\varphi_{2}^{2}\right)^{3}}{8 M_{\mathrm{pl}}^{2}}\left[\frac{6 \varphi_{1}}{\varphi_{1}^{2}+\varphi_{2}^{2}}+\frac{c_{2} \varphi_{1}}{M_{\mathrm{pl}}^{2}}\left\{\frac{6\left(\varphi_{1}^{2}-\varphi_{2}^{2}\right)}{\varphi_{1}^{2}+\varphi_{2}^{2}}+2-2 \frac{\varphi_{1} \varphi_{2}}{\Lambda^{2}}\right\} \cos \left(\frac{\varphi_{1}^{2}+\varphi_{2}^{2}}{2 \Lambda^{2}}\right)\right. \\
& \left.-c_{2} \frac{\varphi_{1}}{M_{\mathrm{pl}}^{2}}\left\{\frac{12 \varphi_{1} \varphi_{2}}{\varphi_{1}^{2}+\varphi_{2}^{2}}+2 \frac{\varphi_{2}}{\varphi_{1}}+\frac{\varphi_{1}^{2}-\varphi_{2}^{2}}{\Lambda^{2}}\right\} \sin \left(\frac{\varphi_{1}^{2}+\varphi_{2}^{2}}{2 \Lambda^{2}}\right)\right], \\
\frac{\partial V}{\partial \varphi_{2}} & =\frac{c_{1}\left(\varphi_{1}^{2}+\varphi_{2}^{2}\right)^{3}}{8 M_{\mathrm{pl}}^{2}}\left[\frac{6 \varphi_{2}}{\varphi_{1}^{2}+\varphi_{2}^{2}}+\frac{c_{2} \varphi_{2}}{M_{\mathrm{pl}}^{2}}\left\{\frac{6\left(\varphi_{1}^{2}-\varphi_{2}^{2}\right)}{\varphi_{1}^{2}+\varphi_{2}^{2}}-2-2 \frac{\varphi_{1} \varphi_{2}}{\Lambda^{2}}\right\} \cos \left(\frac{\varphi_{1}^{2}+\varphi_{2}^{2}}{2 \Lambda^{2}}\right)\right. \\
& \left.-c_{2} \frac{\varphi_{2}}{M_{\mathrm{pl}}^{2}}\left\{\frac{12 \varphi_{1} \varphi_{2}}{\varphi_{1}^{2}+\varphi_{2}^{2}}+2 \frac{\varphi_{1}}{\varphi_{2}}+\frac{\varphi_{1}^{2}-\varphi_{2}^{2}}{\Lambda^{2}}\right\} \sin \left(\frac{\varphi_{1}^{2}+\varphi_{2}^{2}}{2 \Lambda^{2}}\right)\right] .
\end{aligned}
$$

In the right panel of Fig. 2, we show an example for the evolution of the inflaton in the $\left(\frac{\varphi_{1}}{\sqrt{2} M_{\mathrm{pl}}}, \frac{\varphi_{2}}{\sqrt{2} M_{\mathrm{pl}}}\right)$ plane. In this calculation, we assume that $\varphi_{1,2}$ initially stay at the local minimum. The figure shows that the field $a$ evolves aperiodic circle. Along this trajectory, the value of $a$ changes by an amount larger than the Lyth bound [24] during the small change of $\varphi$ in the sub-Planckian range. ¿From this figure, we find that the single inflaton scenario could be realized in this model as long as the conditions mentioned above are satisfied and the fields $\varphi_{1,2}$ start to evolve from a local minimum. 
Next, in order to see the feature of the inflation induced by this field $a$, we calculate the quantities which characterize inflation, that is, the e-foldings $N$, the spectral index $n_{s}$, the tensor-to-scalar ratio $r$ and so on. The change of the inflaton $a$ from some period to the end of inflation can be expressed by using $\varphi$ as

$$
a_{e}-a=-\int_{\varphi}^{\varphi_{e}} \frac{\tilde{\varphi}^{2}}{2 m \Lambda^{2}} d \tilde{\varphi}=\frac{1}{6 m \Lambda^{2}}\left(\varphi^{3}-\varphi_{e}^{3}\right),
$$

where we use eq. (9) under the assumption $\varphi \gg \Lambda$. A value of $\varphi$ at the end of inflation is expressed by $\varphi_{e}$. For the convenience, we may redefine the canonically normalized new inflaton as

$$
\chi \equiv a_{e}+\frac{\varphi_{e}^{3}}{6 m \Lambda^{2}}-a=\frac{\varphi^{3}}{6 m \Lambda^{2}} .
$$

This expression explicitly shows that Sub-Planckian values of $\varphi$ could be enhanced by $\frac{\varphi^{2}}{6 m \Lambda^{2}}$ to result in trans-Planckian values of the inflaton $\chi$. The e-foldings induced by the inflaton change from $\chi$ to $\chi_{e}$ is estimated as

$$
\begin{aligned}
& N=-\frac{1}{M_{\mathrm{pl}}^{2}} \int_{\chi}^{\chi_{e}} d \chi \frac{V}{V^{\prime}} \equiv N(\chi)-N\left(\chi_{e}\right) \\
& N(\chi)=\frac{1}{6 m^{2} n}\left(\frac{M_{\mathrm{pl}}}{\Lambda}\right)^{4}\left(\frac{\varphi}{\sqrt{2} M_{\mathrm{pl}}}\right)^{6}\left[1+\frac{6 c_{2} m}{n(3+m)}\left(\frac{\varphi}{\sqrt{2} M_{\mathrm{pl}}}\right)^{2 m}\right. \\
&\left.\quad \times F\left(1, \frac{3}{m}+1, \frac{3}{m}+2,2 c_{2}\left(1+\frac{m}{n}\right)\left(\frac{\varphi}{\sqrt{2} M_{\mathrm{pl}}}\right)^{2 m}\right)\right],
\end{aligned}
$$

where $V^{\prime}=\frac{d V}{d \chi}$ and $F$ is the hypergeometric function. $\chi_{e}$ is fixed as a value at the end of inflation. In the expression of $N(\chi)$, it might be approximated by the first term since the second term is negligibly small compared with it. However, it should be noted that $N(\chi) \gg N\left(\chi_{e}\right)$ is not satisfied in this scenario.

Slow-roll parameters [25] are easily calculated by using eqs.(77) and (9). We find that they are given by using the model parameters as

$$
\begin{aligned}
\varepsilon & \equiv \frac{M_{\mathrm{pl}}^{2}}{2}\left(\frac{V^{\prime}}{V}\right)^{2}=m^{2}\left(\frac{\sqrt{2} M_{\mathrm{pl}}}{\varphi}\right)^{6}\left(\frac{\Lambda}{M_{\mathrm{pl}}}\right)^{4}\left[\frac{n-2 c_{2}(m+n)\left(\frac{\varphi}{\sqrt{2} M_{\mathrm{pl}}}\right)^{2 m}}{1-2 c_{2}\left(\frac{\varphi}{\sqrt{2} M_{\mathrm{pl}}}\right)^{2 m}}\right]^{2}, \\
\eta & \equiv M_{\mathrm{pl}}^{2}\left(\frac{V^{\prime \prime}}{V}\right) \\
& =m^{2}\left(\frac{\sqrt{2} M_{\mathrm{pl}}}{\varphi}\right)^{6}\left(\frac{\Lambda}{M_{\mathrm{pl}}}\right)^{4} \frac{n(2 n-3)-2 c_{2}(m+n)(2 m+2 n-3)\left(\frac{\varphi}{\sqrt{2} M_{\mathrm{pl}}}\right)^{2 m}}{1-2 c_{2}\left(\frac{\varphi}{\sqrt{2} M_{\mathrm{pl}}}\right)^{2 m}} .
\end{aligned}
$$


If $c_{2}$ terms are neglected in these formulas, we find that these slow-roll parameters at the period characterized by the inflaton value $\chi$ can be represented in the very simple forms such as $\varepsilon \simeq \frac{n}{6\left(N+N\left(\chi_{e}\right)\right)}$ and $\eta \simeq \frac{2 n-3}{6\left(N+N\left(\chi_{e}\right)\right)}$ by using the e-foldings $N$ given in eq. (16). We note that the explicit $m$ dependence in these quantities remains only in the expression of the e-foldings $N$. The end of inflation could occur much before the time when $\varepsilon=1$ is realized. It is crucial to guarantee the field evolution along the local potential minimum and the $c_{2}$ term plays a key role there.

We clarify this feature through a simple observation. When the kinetic energy is equal to the local potential barrier $V_{b}$ which is given by the cos term of eq. (7), the inflaton could go over the potential barrier to the global minimum. The condition could be expressed as $\frac{1}{2} \dot{\chi}^{2} \sim V^{\prime}$. If we use the slow-roll approximation $3 H \dot{\chi}=-V^{\prime}$ here, this condition can be written as $\varepsilon \sim \frac{3 V_{b}}{V}$. Since $V>V_{b}$ is supposed in the model, the end of inflation occurs at the time when $\varepsilon<1$ is satisfied. By solving this condition, we can estimate the value of $\varphi_{e}$ as

$$
\frac{\varphi_{e}}{\sqrt{2} M_{\mathrm{pl}}} \simeq\left(\frac{m^{2} n}{6 c_{2}}\right)^{\frac{1}{2 m+6}}\left(\frac{\Lambda}{M_{\mathrm{pl}}}\right)^{\frac{2}{m+3}}
$$

where we neglect the contribution from the $c_{2}$ terms. In that case, $N\left(\chi_{e}\right)$ has a substantial contribution to determine the e-foldings $N$. To confirm this behavior and estimate the value of $\chi_{e}$, we use the numerical solutions of the field equations (12) which contain the effect of the $c_{2}$ term. The numerical results given in the latter part show a good agreement with the values of $\chi_{e}$ derived by using eq. (18). It supports our picture for the end of inflation.

The spectrum of scalar perturbation predicted by the inflation is expressed as [25]

$$
\mathcal{P}_{\mathcal{R}}(k)=\Delta_{\mathcal{R}}^{2}\left(\frac{k}{k^{*}}\right)^{n_{s}-1}, \quad \Delta_{\mathcal{R}}^{2}=\left.\frac{V}{24 \pi^{2} M_{\mathrm{pl}}^{4} \varepsilon}\right|_{k^{*}} .
$$

The CMB observations give the normalization such that $\Delta_{\mathcal{R}}^{2} \simeq 2.43 \times 10^{-9}$ at $k_{*}=$ $0.002 \mathrm{Mpc}^{-1}$. This constrains the value of $V / \varepsilon$ at the time when the scale characterized by the wave number $k_{*}$ exits the horizon. On the other hand, the remaining e-foldings $N_{*}$ of the inflation after the scale $k_{*}$ exits the horizon is dependent on the reheating phenomena and others as [25]

$$
N_{*} \simeq 61.4-\ln \frac{k_{*}}{a_{0} H_{0}}-\ln \frac{10^{16} \mathrm{GeV}}{V_{k_{*}}^{1 / 4}}+\ln \frac{V_{k_{*}}^{1 / 4}}{V_{\mathrm{end}}^{1 / 4}}-\frac{1}{3} \ln \frac{V_{\mathrm{end}}^{1 / 4}}{\rho_{\mathrm{reh}}^{1 / 4}} .
$$


Taking account of this uncertainty, $N_{*}$ is usually considered to take a value in the range 50 - 60. Here we also use the values in this range and we represent a value of $\varphi$ which gives the e-foldings $N_{*}$ as $\varphi_{*}$. If we use these notations, the above normalization $\Delta_{\mathcal{R}}^{2}$ is found to have a suitable value for

$$
c_{1}=9.5 \times 10^{-8} \frac{n}{N_{*}}\left(\frac{\sqrt{2} M_{\mathrm{pl}}}{\varphi_{*}}\right)^{2 n},
$$

as long as the $c_{2}$ term in the potential is neglected $\mathrm{d}$ As examples, if we suppose $n=3$ and $N_{*}=60, c_{1} \simeq 3 \times 10^{-7}$ and 0.3 are required for $\frac{\varphi_{*}}{\sqrt{2}}=0.5 M_{\mathrm{pl}}$ and $0.05 M_{\mathrm{pl}}$, respectively.

The scalar spectrum index $n_{s}$ and the ratio of the tensor perturbation to the scalar perturbation $r$ can be represented by using the slow-roll parameters $\varepsilon$ and $\eta$ as follows [25],

$$
n_{s}=1-6 \varepsilon+2 \eta, \quad r=16 \varepsilon .
$$

If we use the formulas (17), we can estimate $n_{s}$ and $r$ at $k_{*}$ in this model. In particular, when $c_{2}$ terms are negligibly small, these are summarized by using the e-foldings $N$ in the very simple forms such as

$$
n_{s}=1-\frac{n+3}{3\left(N_{*}+N\left(\chi_{e}\right)\right)}, \quad r=\frac{8 n}{3\left(N_{*}+N\left(\chi_{e}\right)\right)} .
$$

It is very interesting that both expressions of $n_{s}$ and $r$ given in eq. (23) reduce to the same forms which are obtained in the chaotic inflation with the quadratic potential in the case $n=3$ as suggested in [23. They are known to be favored for reasonable $N_{*}$ values such as $N_{*}=50-60$ by BICEP2 results. However, $N\left(\chi_{e}\right) \simeq 0$ is not guaranteed in the present model as mentioned before. As a result, the values of $n_{s}$ and $r$ obtained only for $N_{*}>60$ in the quadratic chaotic inflation model could be realized even for $N_{*}=50-60$. This clarifies a typical feature of the inflation induced in the model. It is induced by the $c_{2}$ term in the potential. As commented above, the trajectory cannot follow the potential minimum and the field suddenly rolls down towards the global minimum as long as the $c_{2}$ term is neglected. Thus, in order to estimate these parameters including the value of $N\left(\chi_{e}\right)$, we need the analysis keeping the effect of $c_{2}$ terms as an indispensable one $\mathrm{e}$

\footnotetext{
${ }^{\mathrm{d}}$ One might find that this condition could be easily satisfied even for a large value of $c_{1}$ near $O(1)$. In fact, if we scale $c_{1}, c_{2}$ and $\Lambda$ such as $x^{2 n} c_{1}, x^{2 m} c_{2}$ and $x^{-1} \Lambda$ with $x$, the potential keeps its form for the scaled $x^{-1} \varphi$. Although the numerical detail in the field evolution has subtle behavior, the basic feature is understood in this way as found in the solution given in Table 1.

${ }^{\mathrm{e}}$ Here we confine our analysis to the case defined by $n=3$ and $m=1$, although other values of $n$ could give interesting results. Those detailed results will be presented elsewhere.
} 


\begin{tabular}{c|cccc|cccc}
\hline & $c_{1}$ & $c_{2}$ & $\frac{\Lambda}{M_{\mathrm{pl}}}$ & $\frac{\varphi_{*}}{\sqrt{2} M_{\mathrm{pl}}}$ & $H_{*}(\mathrm{GeV})$ & $N_{*}$ & $n_{s}$ & $r$ \\
\hline \hline A & $1.66 \times 10^{-6}$ & 0.7 & 0.04 & 0.378 & $0.871 \times 10^{14}$ & 59.0 & 0.971 & 0.107 \\
& $2.04 \times 10^{-6}$ & 0.7 & 0.04 & 0.371 & $0.921 \times 10^{14}$ & 54.2 & 0.968 & 0.119 \\
& $2.42 \times 10^{-6}$ & 0.7 & 0.04 & 0.366 & $0.965 \times 10^{14}$ & 49.1 & 0.965 & 0.131 \\
\hline \multirow{2}{*}{ B } & 0.257 & 6.0 & 0.002 & 0.0512 & $0.945 \times 10^{14}$ & 60.4 & 0.969 & 0.124 \\
& 0.305 & 6.0 & 0.002 & 0.0505 & $0.986 \times 10^{14}$ & 55.0 & 0.966 & 0.136 \\
& 0.364 & 6.0 & 0.002 & 0.0498 & $1.03 \times 10^{14}$ & 50.0 & 0.962 & 0.149 \\
\hline
\end{tabular}

Table. 1 Hubble parameter, spectral index and the tensor-to-scalar ratio for typical examples of four parameters of the model defined by $n=3$ and $m=1$. These model parameters are fixed to realize the observed value for the scalar perturbation amplitude $\Delta_{\mathcal{R}}^{2}$.

In Table 1 we show typical examples which are calculated numerically for different values for the model parameters $c_{1}, c_{2}$ and $\Lambda$. These examples suggest that sufficiently large e-foldings such as $N_{*}=50$ - 60 could be realized as long as $\Lambda \ll \varphi_{*}$ is satisfied even for the sub-Planckian inflaton value $\varphi_{*}<M_{\mathrm{pl}}$ The Hubble parameter during the inflation takes values around $10^{14} \mathrm{GeV}$ as shown in this table. The predicted values of $n_{s}$ and $r$ are also listed in each case. In this calculation, we again use the solutions obtained from the field equations (12).

In Fig. 3, we plot the predicted points in the $\left(n_{s}, r\right)$ plane for $N_{*}=50-60$ in the cases $\mathrm{A}$ and $\mathrm{B}$ given in Table 1. Although the model parameters $c_{2}$ and $\Lambda$ are required to take values in suitable regions to realize the observational data, the serious fine tuning of parameters seems not to be necessary. As a reference, we plot the prediction of the $\phi^{2}$ chaotic inflation as a dotted line in the same figure. Two crosses on it show the predicted points for $N_{*}=50$ and 60 . The figure shows that this model could realize the $\left(n_{s}, r\right)$ points in the wider ranges which cannot be reached for $N_{*}=50-60$ in the $\phi^{2}$ chaotic inflation scenario. It should be noted that that such points are realized even for the values of $N_{*}$ such as 50 - 60 by changing the model parameters suitably. We find that the points of the case B somewhat shift from the line for the quadratic chaotic model. This can

\footnotetext{
${ }^{\mathrm{f}}$ The values of $N_{*}$ in Table 1 are obtained through the direct numerical integration of Hubble parameters. If we use the formula given in eq. (16), the similar value can be obtained within a few percent difference from those. Both $n_{s}$ and $r$ are found to be not sensitive for such differences.
} 
be easily understood. As found from eq. (17), the $c_{2}$ term makes $\eta$ somewhat smaller than $\varepsilon$ compared with the $\phi^{2}$ chaotic inflation model where $\varepsilon=\eta$ is satisfied. Since this effect becomes larger for the parameters in the case $\mathrm{A}$ than the ones in the case B, the predicted points appear below the line for the $\phi^{2}$ chaotic inflation model. We find that the model is expected to predict $\left(n_{s}, r\right)$ in the region on or below the line predicted by the $\phi^{2}$ chaotic inflation model and also in the region where $n_{s}$ takes a larger value than the one predicted by the $\phi^{2}$ chaotic inflation model for the fixed $N_{*}$ value. These features show that the model could be an alternative interesting scenario to the simple $\phi^{2}$ chaotic inflation model. If both values of $n_{s}$ and $r$ could be constrained through the precise data obtained from the future CMB observations, the model could be tested in the near future.

Finally, we should address the notorious $\eta$-problem in the model. Although we could make higher order terms suppressed by the Planck scale in the potential ineffective, the $\eta$ problem still remains in the model. It may be formulated in two forms such that, (i) why $\frac{\Lambda}{M_{\mathrm{pl}}}$ in eq. (7) is smaller than $O(1)$, and (ii) why $\tilde{m}_{S}^{2}, m_{S}^{2}$ and $\kappa_{1} \varphi^{2}$ are small enough in comparison with $H^{2}$. As mentioned before, the first one is closely related to the UV completion of the model, which fixes the exponential part of the potential. This could be solved only if the UV completion is found and its dynamics is clarified. It is beyond the present scope. The second one might be also determined in the UV completed model. In the present model, however, the values of $\tilde{m}_{S}^{2}$ and $m_{S}^{2}$ are related to other low energy physics, that is, the neutrino masses. This additional aspect might give a new physical meaning to the $\eta$ problem in this model. We might approach the problem based on this view point.

\subsection{Reheating after the end of inflation}

The result shown in the previous part suggests that the model has favorable features as an inflation scenario. In order for the model to be a realistic one, it is required that the inflaton energy should be transferred to radiation energy to reheat the Universe after the end of inflation. It could be expected to occur if the aperiodic circular motion of the inflaton stops at a certain period and starts to behave as matter through the oscillation around the global minimum of the potential. In fact, such a behavior can be found to occur in the right panel of Fig. 2. Since the kinetic energy of the fields becomes larger compared with the local potential barrier which gradually becomes smaller, the field 


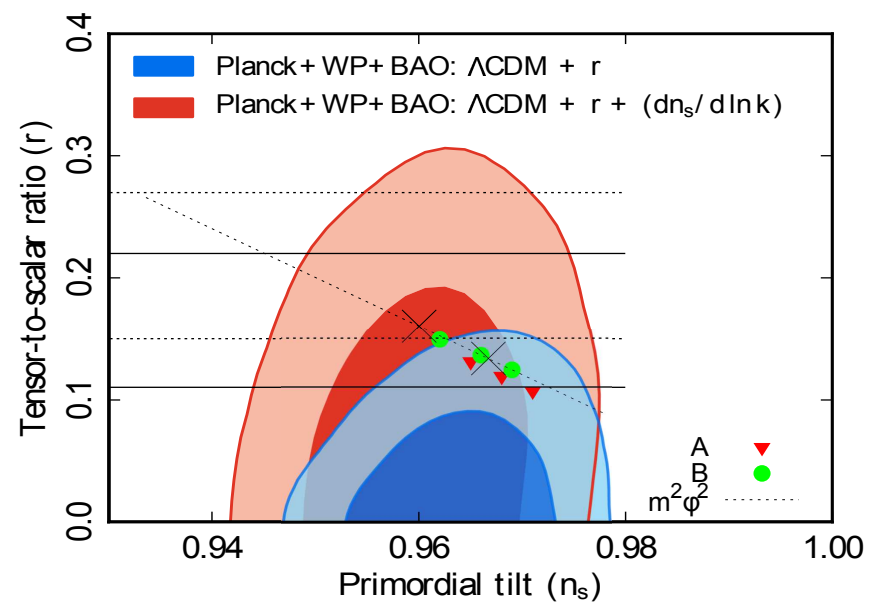

Fig. 3 Predicted values of $\left(n_{s}, r\right)$ for several parameter sets $\left(c_{2}, \frac{\Lambda}{M_{\mathrm{pl}}}\right)$ given in Table 1 . A dotted line represents the prediction by the quadratic chaotic inflation model and the crosses correspond to the points for $N_{*}=50$ and 60. Horizontal solid lines and dotted lines represent the Bicep2 $1 \sigma$ constraints with and without the foreground subtraction, respectively [17. Contours given as Fig. 4 in Planck Collaboration XXII [16] are used here. Since the running of the spectral index $n_{s}$ is negligible in the present model, the blue contours should be compared with the predictions.

component $\varphi$ is expected to leave the local minimum and go over the potential toward the global minimum at a certain period.

As reheating processes during the $\varphi_{1,2}$ oscillation, we have to consider both preheating due to the parametric resonance [26] through quartic interactions of $S$ with $\phi$ and $\eta$ and also the perturbative decay due to an interaction term $\mu S \eta^{\dagger} \phi$ given in eq. (21). Just after the end of inflation, the fields $\varphi_{1,2}$ start the oscillation around the global minimum with very large amplitude. Since the fields coupled with them have large effective masses and then it seems difficult for $\varphi_{1,2}$ to produce these particles. However, the particle production due to the parametric resonance is known to occur effectively even in such a situation. In this model the parametric resonance due to the scalar quartic couplings might realize rather high reheating temperature.

On the other hand, only preheating cannot transfer the inflaton energy to the radiation completely [26, 27]. The decay of $\varphi_{1,2}$ induced through three scalars interaction such as $\frac{\mu}{\sqrt{2}} \varphi_{1} \eta^{\dagger} \phi$ and $\frac{i \mu}{\sqrt{2}} \varphi_{2} \eta^{\dagger} \phi$ can complete the energy transfer in this model. The decay width for these processes is estimated as $\Gamma_{\varphi_{i}}=\frac{1}{8 \pi} \frac{|\mu|^{2}}{\bar{m}_{i}}$ where $\bar{m}_{1}^{2}=\tilde{m}_{S}^{2}+m_{S}^{2}$ and $\bar{m}_{2}^{2}=\tilde{m}_{S}^{2}-m_{S}^{2}$. Since $\tilde{m}_{S} \gg m_{S}$ is assumed to be satisfied here, the reheating temperature realized 
through these processes could be estimated as [27]

$$
T_{R} \simeq 0.35 g_{*}^{-1 / 4}|\mu| \sqrt{\frac{M_{\mathrm{pl}}}{\tilde{m}_{S}}},
$$

where we use $g_{*}=116$ as the relativistic degrees of freedom in this model. Since both $\mu$ and $\tilde{m}_{S}$ are relevant to the neutrino mass generation as shown in the previous part, we should take account of the constraint from it. The present inflation scenario also requires that eq. (17) is the dominant potential of $S$ at the inflation era. This brings about the additional constraints on $\tilde{m}_{S}$ as

$$
\tilde{m}_{S} \ll \sqrt{c_{1}}\left(\frac{\varphi_{*}}{M_{\mathrm{pl}}}\right)^{n-2} \varphi_{*} \simeq 3.1 \times 10^{-4}\left(\frac{n}{N_{*}}\right)^{1 / 2}\left(\frac{M_{\mathrm{pl}}}{\varphi_{*}}\right)^{2} \varphi_{*},
$$

where eq. (21) is used. If we apply $N_{*}=60$ and $\varphi_{*} \simeq 0.5 M_{\mathrm{pl}}$ which are the typical values for the case $n=3$ in the previous part, the bound for $\tilde{m}_{S}$ can be obtained as $\tilde{m}_{S} \ll 3.4 \times 10^{14} \mathrm{GeV}$. Taking account of this constraint, we may estimate the reheating temperature through this process as

$$
T_{R} \simeq 1.6 \times 10^{8}\left(\frac{\left|\lambda_{5}\right|}{10^{-6}}\right)^{1 / 2}\left(\frac{\tilde{m}_{S}}{m_{S}}\right)\left(\frac{\tilde{m}_{S}}{10^{6} \mathrm{GeV}}\right)^{1 / 2} \mathrm{GeV} .
$$

Here we also note that $\left|\lambda_{5}\right|$ should be smaller than $O\left(10^{-6}\right)$ from the present bound of DM direct search since the lightest neutral component of $\eta$ is DM and its mass is $\sim 1 \mathrm{TeV}$ [14. We find that the reheating temperature could be in a rather wide range such as $10^{5} \mathrm{GeV} \lesssim T_{R} \lesssim 10^{15} \mathrm{GeV}$ depending on a value of $\tilde{m}_{S}$. This temperature is high enough to produce thermal right-handed neutrinos in the present model since the masses of righthanded neutrinos are assumed to be of $O(1) \mathrm{TeV}$. If the right-handed neutrino masses are sufficiently degenerate, the baryon number asymmetry could be generated through the resonant leptogenesis as discussed in [14]. Right-handed neutrinos need not to be light but they could have large mass such as $O\left(10^{9}\right) \mathrm{GeV}$ in a consistent way with this neutrino mass model [14]. Even in that case, eq. (26) shows that sufficient reheating temperature could be induced for leptogenesis to work well without the resonant effect. Anyway, the model could cause sufficient reheating temperature for the generation of baryon number asymmetry independently from the details of preheating in the model. 


\section{Summary}

In this paper we have considered an extension of the radiative seesaw model with a complex singlet scalar to realize the inflation of the Universe keeping favorable features of the original model, that is, the simultaneous explanation of the small neutrino masses, the DM abundance and the baryon number asymmetry in the Universe. This singlet scalar plays a crucial role not only in the exponential expansion of the Universe as an inflaton but also in the small neutrino mass generation. In this scenario inflaton trajectory follows an aperiodic circle during the inflation. This feature makes it possible that sub-Planckian values of the relevant field induce trans-Planckian changes of the inflaton value which is needed for the sufficient e-foldings. The model could be free from the serious problem caused by trans-Planckian field values. However, the $\eta$ problem still remains. The UV completion of the model is expected to give a solution for it.

We have also shown that the model has other interesting aspects as the inflation model. As a limiting situation, it gives the same formulas for the spectral index $n_{s}$ and the tensorto-scalar ratio $r$ as ones of the $m^{2} \varphi^{2}$ type chaotic inflation, in which $r$ could take a large value. In more general cases, we have estimated them by solving numerically the field equations for the component fields of the singlet scalar. The tensor-to-scalar ratio could take large values in these cases also. Both the spectral index $n_{s}$ and the tensor-to-scalar ratio $r$ could have values which are favorable from the recent $\mathrm{CMB}$ observations. If the precise data from the CMB observations are given in the near future, we could restrict the model parameters much more. Since the roughly estimated reheating temperature tells us that it could be high enough for leptogenesis, the model seems to explain consistently the crucial problems in the SM including the baryon number asymmetry. Although we cannot mention the origin of the specific potential at this stage, the features shown by the model seem to be interesting. The model may deserve further study.

\section{Acknowledgements}

R. H. S. Budhi is supported by the Directorate General of Higher Education (DGHE) of Indonesia (Grant Number 1245/E4.4/K/2012). S. K. is supported by Grant-in-Aid for JSPS fellows (Grant Number 26.5862). D. S. is supported by JSPS Grant-in-Aid for Scientific Research (C) (Grant Number 24540263) and MEXT Grant-in-Aid for Scientific 
Research on Innovative Areas (Grant Number 26104009). 


\section{References}

[1] ATLAS Collaboration, G. Aad, el al., Phys. Lett. B716 (2012) 1; CMS Collaboration, S. Chatrchyan, el al., Phys. Lett. B716 (2012) 30.

[2] Super-Kamiokande Collaboration, Y. Fukuda, et al., Phys. Rev. Lett. 81 (1998) 1562; SNO Collaboration, Q. R .Ahmad, et al., Phys. Rev. Lett. 89 (2002) 011301; KamLAND Collaboration, K. Eguchi, et al., Phys. Rev. Lett. 90 (2003) 021802; K2K Collaboration, M. H. Ahn, et al., Phys. Rev. Lett. 90 (2003) 041801;

[3] T2K Collaboration, K. Abe, et al., Phys. Rev. Lett. 107 (2011) 041801; Double Chooz Collaboration, Y. Abe, et al., Phys. Rev. Lett. 108 (2012) 131801; RENO Collaboration, J. K. Ahn, et al., Phys. Rev. Lett. 108 (2012) 191802; The Daya Bay Collaboration, F. E. An, et al., Phys. Rev. Lett. 108 (2012) 171803.

[4] WMAP Collaboration, D. N. Spergel, et al., Astrophys. J. 148 (2003) 175; SDSS Collaboration, M. Tegmark, et al., Phys. Rev. D69 (2004) 103501.

[5] A. Riotto and M. Trodden, Ann. Rev. Nucl. Part. Sci. 49 (1999) 35; W. Bernreuther, Lect. Notes Phys. 591 (2002) 237; M. Dine and A. Kusenko, Rev. Mod. Phys. 76 (2003) 1 .

[6] R. Barbieri, L. J. Hall and V. S. Rychkov, Phys. Rev. D74 (2006) 015007.

[7] E. Ma, arXiv:0810.5574.

[8] C. T. Hill, Phys. Rev. D89 (2014) 073003.

[9] E. Ma, Phys. Rev. D73 (2006) 077301.

[10] J. Kubo, E. Ma and D. Suematsu, Phys. Lett. B642 (2006) 18; J. Kubo and D. Suematsu, Phys. Lett. B643 (2006) 336; D. Suematsu, Eur. Phys. J. C56 (2008) 379; D. Aristizabal Sierra, J. Kubo, D. Restrepo, D. Suematsu and O. Zapata, Phys. Rev. D79 (2009) 013011; D. Suematsu, T. Toma and T. Yoshida, Phys. Rev. D79 (2009) 093004; D. Suematsu, T. Toma and T. Yoshida, Phys. Rev. D82 (2010) 013012; D. Suematsu, Eur. Phys. J C72 (2012) 1951.

[11] T. Hambye, F.-S. Ling, L. L. Honorez and J. Roche, JHEP 07 (2009) 090. 
[12] E. Ma, Annales Fond. Broglie 31 (2006) 285; H. Fukuoka, J. Kubo and D. Suematsu, Phys. Lett. B678 (2009) 401; D. Suematsu and T. Toma, Nucl. Phys. B847 (2011) 567; H. Fukuoka, D. Suematsu and T. Toma, JCAP 07 (2011) 001; H. Higashi, T. Ishima and D. Suematsu, Int. J. Mod. Phys. A26 (2011) 995.

[13] D. Suematsu, Phys. Rev. D85 (2012) 073008.

[14] S. Kashiwase and D. Suematsu, Phys. Rev. D86 (2012) 053001; S. Kashiwase and D. Suematsu, Eur Phys. J C73 (2013) 2484.

[15] M. Flanz, E. A. Pascos and U. Sarkar, Phys. Lett. B345 (1995) 248; L. Covi, E. Roulet and F. Vissani, Phys. Lett. B384 (1996) 169; A. Pilaftsis, Phys. ReV. D56 (1997) 5431.

[16] Planck Collaboration, P. A. R. Ade, et al., arXiv1303.5082 [astro-ph.CO].

[17] BICEP2 Collaboration, P.A.R Ade, et al, Phys. Rev. Lett. 112 (2014) 241101.

[18] H. Murayama, H. Suzuki, T. Yanagida and J. Yokoyama, Phys. Rev. Lett. 70 (1993) 1912; D. Suematsu and Y. Yamagishi, Mod. Phys. Lett. A10 (1995) 2923; H. Murayama, K. Nakayama, F. Takahashi and T. T. Yanagida, arXiv1404.3857.

[19] F. L. Bezrukov and M. Shaposhnikov, Phys. Lett. B659 (2008) 703.

[20] K. Freese, J. A. Frieman and A. V. Olinto, Phys, Rev. Lett. 65 (1990) 3233.

[21] J.-O. Gong and H. M. Lee, JHEP 04 (2012) 128; S. Kanemura, T. Matsui and T. Nabeshima, Phys. Lett. B723 (2013) 126.

[22] R. N. Lerner and J. McDonald, Phys.Rev. D80 (2009) 123507; R. N. Lerner and J. McDonald, Phys. Rev. D83 (2011) 123522; R. N. Lerner and J. McDonald, JCAP 11 (2012) 019.

[23] J. McDonald, arXiv:1404.4620 [hep-ph].

[24] D. H. Lyth, Phys. Rev. Lett. 78 (1997) 1861.

[25] For reviews, D. H. Lyth and A. Riotto, Phys. Rep. 314 (1999) 1; A. R. Liddle and D. H. Lyth, Cosmological Inflation and Large-Scale Structure (Cambridge, 2000). 
[26] L. Kofman, A. Linde and A. A. Starobinsky, Phys. Rev. Lett. 73 (1994) 3195; L. Kofman, A. Linde and A. A. Starobinsky, Phys. Rev. D56 (1997) 3258.

[27] For a recent review, R. Allahverdi, R. Brandenberger, F.-Y. Cyr-Racine and A. Mazumdar, arXiv:1001.2600 [hep-th], and see references therein. 